better after the firm foundation given by tho present syllabuses. This would certainly be true of the good physics student who would have no difficulty with the transition, but the weaker brethren might not find it quite so easy. In connexion with this it is perhaps relevant to point out that at least one of the British universities sets alternative questions based on M.K.S. units in the magnetism and electricity paper, while, of course, the system is widely used in the United States.

Reference is also made in the book to the discussion going on concerning the introduction of more modern topics into the advanced syllabus, and Dr. Brown, very wisely in my own opinion, warns against the inclusion of any such topics if they are allowed to crowd out the more basic parts of the subject. He agrees that all students should know something about modern developments in pure and applied physics, and this belief would be shared by most people; any change, however, would have to be thought out carefully. To stimulate interest among students in the newer work and to serve as an introduction to further reading, two chapters at the end of the book deal with electronics, radioactivity and atomic structures.

This is an excellent and well-written work, and, as one would expect from such an experienced author, a book from which a student should derive great help.

T. M. YARWOOD

\section{METALLURGY OF 55 UNCOMMON METALS}

\section{Rare Metals Handbook}

Edited by Clifford A. Hampel. Second edition. Pp. xvi 715. (New York: Reinhold Publishing Corporation; London: Chapman and Hall, Itd., 1961.) 160s.

$\mathrm{T}$

HE first edition of this hand-book set out existing reference data on thirty-four elements which at that time (1954) were not available in large quantities or were too expensive to warrant wide usago. In the intervening years, many of those originally referred to as 'rare' have becomo more plentiful in supply and more widely used in industry and laboratory. In this second edition (1961) additions have been made, (chiefly from the rare earth group) and chapters formerly devoted to two metals have warranted separate treatment.

Inevitably, the only method of compilation in a volume of this type is to use specialists in each field and an editor of broad outlook. The result more than justifies the new edition, and this book will be invaluable to all metallurgical libraries, whether concerned with manufacturing processes, development, or research.

In addition to thirty-three separate chapters on individual metals or groups from the periodic system, there is a general review in Chapter 1 on occurrence and processing problems, and a final chapter summarizing physical properties in a form easy to consult, with metals not covered in the hand-book included for reference. Many tables in this chapter show an order of merit in addition to the usual alphabetical list, a fashion which might be more widely adopted in similar reference books.

Each chapter on separate metals gives a full treatment on occurrence, preparation, refining where applicable, propertios and applications, with many references for further exploration. Information on such metals as beryllium, germanium and silicon is still obviously affected by commercial security, but extensive publications in recent years on uranium, plutonium and titanium are more fully covered. I was somewhat amused to find niobium described under columbium, and a quarter page of text devoted to an explanation of why the American name has been adopted. The inclusion of metals of the rare earth group is most welcome. During the past few years work in this field has been mainly concentrated in the United States and U.S.S.R. and there is no doubt that interest in these metals is bound to increase with improved methods of separation, refining and fabrication.

This book utilizes the two-column format, which suits a reference book, and the text, diagrams and photographs are excellently reproduced. In spite of the price, this is a work of reference which inevit. ably will find its way into every laboratory dealing with materials science.

C. R. Tottre

\section{ANALYTIC FUNCTIONS}

\section{Analytic Functions}

By R. Nevanlinna, H. Behnke and H. Grauert, L. V. Ahlfors, D. C. Spencer, L. Bers, K. Kodaira, M. Heins, J. A. Jenkins. (Princeton Mathematical Series, No. 24.) Pp. vii + 197. (Princeton, N.J.: Princeton University Press; London: Oxford University Press, 1960.) 40s. net.

HIS book contains the principal addresses delivered at the conference on "Analytic Functions" held during September 1957, at Princeton, New Jersey. The authors are all very distinguished, and their articles, which are concerned with the most recent advances in the fields covered, are of a very high standard indeed. In a review of this kind it is impossible to give more than the following brief outlines of the contents.

$R$. Nevanlinna gives a short account of an absolute calculus. $\mathrm{He}$ then proceeds to prove the theorem of Liouville concerned with conformal transformations in Euclidean space of dimension greater than 2.

H. Behnke and H. Grauert contribute a survey article which will be of immense value to those interested in complex analysis of several variables, especially as it contains an extensive bibliography. It deals with attempts to arrive at a suitable analogue of Riemann surfaces. There is considerable dependence on topology of a high degree of sophistication.

Teichmuller showed that the space of Riemann surfaces with generators of genus $g$ greater than unity has a natural metric by which it is topologized as a $6 g-6$ dimensional manifold. L. V. Ahlfors in his article shows that over this topology is a unique complex analytic structure with respect to which the elements of the Riemann matrix are analytic functions.

D. C. Spencer applies a technique that he and Kodaira used earlier to describe the deformation of complex analytic structures to other structures. Particular attention is directed to real foliate structures and to structures defined by pscudogroups in the sense of E. Cartan.

After an introduction to quasi-conformal mapping and Fuchsian groups L. Bers proves a theorem due to Teichmüller. This result, which leads to the natural 